\title{
Incubation time approach to rock dynamic strength characterization
}

\author{
A. Evstifeev ${ }^{1}$, E. Cadoni ${ }^{2}$, and Y. Petrov ${ }^{3}$ \\ 1 St.-Petersburg State University, 199034 St. Petersburg, Russia \\ 2 DynaMat Lab., University of Applied Sciences of Southern Switzerland, 6952 Canobbio, Switzerland \\ ${ }^{3}$ Institute Problem of Mechanical Engineering, Russian Academy of Sciences, 199178 St. Petersburg, Russia
}

\begin{abstract}
The dynamic characterization of rocks under intermediate and high strain rates is fundamental to understand the material behaviour in case of heavy earthquakes and dynamic events. The implementation of material constitutive laws is of capital importance for the numerical simulation of the dynamic processes as those caused by earthquakes. These data are necessary and require experimental techniques able to induce on the rock materials state of loading reproducing the actual dynamic condition. Consider the work [1]. The dynamic characterization has been carried out by means of two special apparatus: the split Hopkinson tension bar and the hydro-pneumatic machine. These equipments are briefly described with a discussion on the results of dynamic tension tests at three different strain rates $(0.1,10,100$ strain/s) on Onsernone Orthogneiss for loading directions $0^{\circ}, 45^{\circ}$ and $90^{\circ}$ with respect to the schistosity. Results of the tests show a significant strain rate sensitive behaviour, exhibiting dynamic tensile strength increasing with strain rate, up to about two times with respect to the quasi-static conditions in the case of $0^{\circ}$ and $45^{\circ}$ orientation and more than three times in the case of $90^{\circ}$ at high strain rates.
\end{abstract}

\section{Introduction}

The dynamic mechanical properties of rocks can be very different from those exhibited in quasi-static conditions. Specific investigations in such dynamic ranges appear necessary to correctly understand their behaviour under intermediate and high strain rate conditions. Particular attention should be addressed to the dynamic tensile strength of rock materials that represent a relevant parameter to many rock mechanics applications.

The dynamic tensile strength can be obtained directly or indirectly by means of different experimental methods based on drop weight machines, split Hopkinson pressure bar (SHPB), gas gun, etc. The direct measurement of the dynamic tensile strength should be preferred because the measurement should be simple enough without introducing complexities such as a superposition of waves or inertia effects. Furthermore, rocks are often anisotropic in nature and their behaviour is strongly influenced by anisotropy [2].

In order to obtain the dynamic properties, the testing methods have to cover a wide spectrum of loading and strain rates. The strain rates achievable by the dynamic devices are typically from $10^{-1}$ to $10^{3} \mathrm{~s}^{-1}$, while ordinary hydraulic testing machines can load specimens at strain rates up to $10^{-3} \mathrm{~s}^{-1}$.

Results from several research activities in rock mechanics are available in literature investigating influence of strain rate on mechanical properties of different types of rocks [3]. It is usually accepted that rocks exhibit higher strength values as strain rate increases; this behaviour is mainly due to microscopic inhomogeneity, affecting principally sensitiveness to medium strain rates, and microcrack formations, failure surfaces development, which influence primarily high strain rates regime.

The direct measurement of the dynamic tensile strength of the Onsernone Gneiss (three strain rates for $0^{\circ}, 45^{\circ}$ and $90^{\circ}$ orientations) has been carried out using the split Hopkinson tension bar (SPTB) and the hydro-pneumatic machine (HPM) installed in the DynaMat Laboratory of the University of Applied Sciences of Southern Switzerland.

\section{Materials}

The rock material used for testing is an Orthogneiss from the Onsernone Valley (Swiss Alps) of the Cantone Ticino (Switzerland). Its petrography is characterized by the presence of white feldspar (albite) and transparent quartz. Also included are a relatively large quantity of white mica (muscovite) and dark mica (biotite) even if this is not evenly distributed. The mean grain size of the crystals is about $0.5 \mathrm{~mm}$ while the maximum grain size is $1 \mathrm{~mm}$. Series of cores $($ diameter $=20 \mathrm{~mm}$, height/diameter $=1$ ) was drilled out in three directions: the first parallel $\left(\theta=0^{\circ}\right)$ and the second perpendicular $\left(\theta=90^{\circ}\right)$ to the plane of schistosity, the third sampling direction oriented at $\theta=45^{\circ}$ to the plane of schistosity. As result $\theta$ is then defined as the angle of schistosity with the loading direction.

The material is characterized by an apparent density of $2^{\prime} 710 \mathrm{~kg} / \mathrm{m}^{3}$ and an absolute density of $2^{\prime} 740 \mathrm{~kg} / \mathrm{m}^{3}$. The Young's modulus at $\theta=0^{\circ}$ is $39.3 \mathrm{GPa}$ and its Poisson's ratio is 0.3 . The compression strength has been evaluated on three samples for each $\theta$ and the mean values are $189 \pm 8$ and $203 \pm 7 \mathrm{MPa}$ for $\theta=0^{\circ}$ and $\theta=90^{\circ}$, respectively. In order to compare dynamic and quasi-static regime directly, excluding the results of any size effect influence, the tensile static strength has been measured on $20 \mathrm{~mm}$ diameter, $20 \mathrm{~mm}$ high specimens (the same geometry of the dynamic tests). Three specimens have been used to evaluate the tensile strength for each $\theta$ and the mean values are 12.6 \pm 0.5 , 6.1 \pm 0.8 and 2.6 $\pm 1.1 \mathrm{MPa}$ for $\theta=0^{\circ}, \theta=45^{\circ}$ and $\theta=90^{\circ}$, respectively. 
Table 1. High strain rate results from [1].

\begin{tabular}{|l|c|c|c|}
\hline ID & $\begin{array}{c}\text { Orientation of } \\
\text { schistosity } \\
\left({ }^{\circ}\right)\end{array}$ & $\begin{array}{c}\text { Dynamic } \\
\text { tensile stress } \\
(\mathrm{MPa})\end{array}$ & $\begin{array}{c}\text { Stress } \\
\text { rate } \\
(\mathrm{GPa} / \mathrm{s})\end{array}$ \\
\hline 0_N1 & 0 & 25.71 & 1.161 \\
\hline 0_N2 & 0 & 24.96 & 1.151 \\
\hline 0_N6 & 0 & 25.70 & 870 \\
\hline 45_N3 & 45 & 8.07 & 405 \\
\hline 45_N4 & 45 & 16.86 & 788 \\
\hline 45_N7 & 45 & 15.65 & 737 \\
\hline 90_N1 & 90 & 6.50 & 257 \\
\hline 90_N4 & 90 & 9.56 & 545 \\
\hline 90_N6 & 90 & 9.96 & 534 \\
\hline
\end{tabular}

\section{Tensile test results}

\subsection{High strain rate tests}

The results of the tests at high strain rates carried out with the Split Hopkinson Tensile Bar equipment are shown in Table 1 . The tests have been performed in tension with the load in three orientations $\theta=0^{\circ}, \theta=45^{\circ}$ and $\theta=90^{\circ}$ with respect to the schistosity plane. The strain rate of the test is dependent on two variables: the behaviour of the material and the loading of the pre-stressed bar. A constancy of the preload has been chosen in order to verify the response of the rock specimen under the same loading condition (in this case a preload of $18 \mathrm{kN}$ ) varying the orientation schistosity.

\subsection{Intermediate strain rate tests}

The intermediate strain rate tests have been carried out using a Hydro Pneumatic Machine with two different calibrated orifices with diameters of 1 and $3 \mathrm{~mm}$. With the lower diameter, the intermediate velocity (about $200 \mathrm{MPa} / \mathrm{s}$ ) is lower than the one obtained (about $1,500 \mathrm{MPa} / \mathrm{s}$ ) with the greater diameter. The results are shown in Table 2 . The tests have been carried out in tension for $\theta=0^{\circ}, \theta=45^{\circ}$ and $\theta=90^{\circ}$.

\section{The concept of incubation time}

Considering phenomena such as brittle failure of a defectfree specimen, fracture of specimen with macro-defect (crack), we deal with strength properties of the materials. These very different processes exhibit some important common features. In the case of slowly applied load there exists the threshold value of load amplitude. In the case of very short durations the strength characteristics of materials are considerably different from those obtained in the case of quasi-static testing. This can be explained
Table 2. Intermediate strain rate results from [1].

\begin{tabular}{|l|c|c|c|}
\hline ID & $\begin{array}{c}\text { Orientation of } \\
\text { schistosity } \\
\left({ }^{\circ}\right)\end{array}$ & $\begin{array}{c}\text { Dynamic } \\
\text { tensile stress } \\
(\mathrm{MPa})\end{array}$ & $\begin{array}{c}\text { Stress } \\
\text { rate } \\
(\mathrm{GPa} / \mathrm{s})\end{array}$ \\
\hline 0_N3 & 0 & 19.78 & 1.752 \\
\hline 0_N4 & 0 & 17.56 & 1.370 \\
\hline 0_N7 & 0 & 14.82 & 1.439 \\
\hline 0_N8 & 0 & 16.88 & 0.185 \\
\hline 0_N9 & 0 & 15.19 & 0.207 \\
\hline 0_N13 & 0 & 16.38 & 0.191 \\
\hline 45_N1 & 45 & 8.66 & 1.401 \\
\hline 45_N2 & 45 & 10.72 & 1.457 \\
\hline 45_N8 & 45 & 8.20 & 1.514 \\
\hline 90_N2 & 90 & 2.26 & 1.665 \\
\hline 90_N3 & 90 & 4.42 & 1.354 \\
\hline 90_N7 & 90 & 5.96 & 1.525 \\
\hline 90_N8 & 90 & 4.56 & 0.201 \\
\hline 90_N9 & 90 & 1.91 & 0.116 \\
\hline 90_N11 & 90 & 4.95 & 0.193 \\
\hline
\end{tabular}

by the circumstance that the time of loading is of the same order as the typical time of certain processes on the micro-level. The above-mentioned time can be described by virtue of the material constant and will be referred to as "incubation time". An efficient criterion for the analysis of brittle fracture for defect-free material can be formulated in the following form [4-7]:

$$
\frac{1}{\tau} \int_{\mathrm{t}-\tau}^{\mathrm{t}} \frac{\sigma(s)}{\sigma_{\mathrm{st}}} \mathrm{ds} \leq 1
$$

where $\tau$ is the incubation time, $\sigma_{\mathrm{st}}$ is a tensile strength for quasi-static loading and $\sigma(t)$ is the applied stress which for $\mathrm{t}<0$ is supposed to be zero. The instant of fracture $t^{*}$ corresponds to the earliest realization of equality in Equation (1) or, in the general case, violation of this condition.

Consider the uniaxial extension of a bar for Constant Stress-Rate loading.

$$
\sigma(\mathrm{t})=\dot{\sigma} \mathrm{tH}(\mathrm{t})
$$

where $\mathrm{H}(\mathrm{t})$ is a Heaviside function, $\dot{\sigma}$ is a Stress rate (supposed to be constant).

For this case we obtain fracture time $\mathrm{t}^{*}$ from equation

$$
\mathrm{t}_{*}^{2}-\left(\mathrm{t}_{*}-\tau\right)^{2} \mathrm{H}\left(\mathrm{t}_{*}-\tau\right)=\frac{2 \tau \sigma_{\mathrm{st}}}{\dot{\sigma}}
$$

End Tensile stress is given by

$$
\sigma_{*}(\mathrm{t})=\sigma\left(\mathrm{t}^{*}\right)
$$


Table 3. Parameters.

\begin{tabular}{|l|c|c|}
\hline $\begin{array}{l}\text { Orientation of } \\
\text { schistosity } \\
\left({ }^{\circ}\right)\end{array}$ & $\begin{array}{c}\text { static tensile } \\
\text { strength } \\
(\mathrm{MPa})\end{array}$ & $\begin{array}{c}\tau \\
(\mu \mathrm{s})\end{array}$ \\
\hline 0 & 12.6 & 44 \\
\hline 45 & 6.1 & 35 \\
\hline 90 & 2.6 & 59 \\
\hline
\end{tabular}

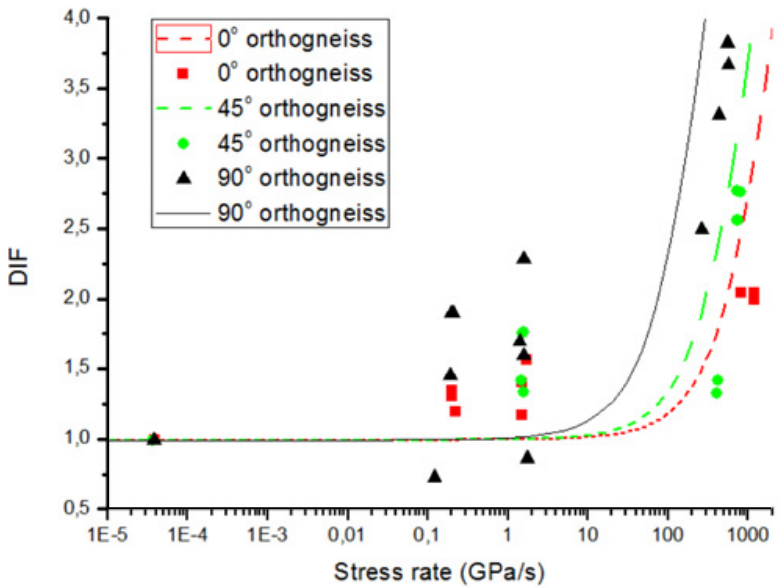

Fig. 1. DIF versus stress rate for the Orthogneiss at $\theta=0^{\circ}$, $\theta=45^{\circ}$ and $\theta=90^{\circ}$.

It follows from Equation (3) that for "rapid" loading we obtain

$$
\sigma_{*}=\left(2 \dot{\sigma} \tau \sigma_{\mathrm{st}}\right)^{1 / 2}, \quad \text { for } \quad \dot{\sigma} \tau \geq 2 \sigma_{\mathrm{st}}
$$

For "slow" loading

$$
\sigma_{*}=\sigma_{\mathrm{st}}+\frac{\sigma \tau}{2}, \quad \text { for } \quad \dot{\sigma}<2 \sigma_{\mathrm{st}}
$$

\subsection{Strain rate effect}

The dynamic effect on the tensile strength can be represented by the ratio between the dynamic and static strength, well known as dynamic increase factor (DIF $=$ $\left.\mathrm{f}_{\mathrm{t} \text {,dynamic }} / \mathrm{f}_{\mathrm{t} \text {,static }}\right)$. Several researchers have found out that DIF of strength increases with strain rates. Use the concept incubation time and construct strain rate curves for different orientation of schistosity. Constants for equation (3) presented in Table 3.

On Fig. 1 shows the effect of the orientation on the dynamic tensile strength for different Orientation of schistosity. Points show the experimental data from Tables 1, 2.

Curves show the theoretical line constructed by the formula (1) with parameters from the Table 3. We can see good compliance theoretical approach and experimental data. Parameter $\sigma_{\text {st }}-$ static tensile stress. It is determines the horizontal section on theoretical curve. Parameter $\tau$-can move theoretical curve to the right or to the left. Theoretical curve was built as a middle line of experimental data.

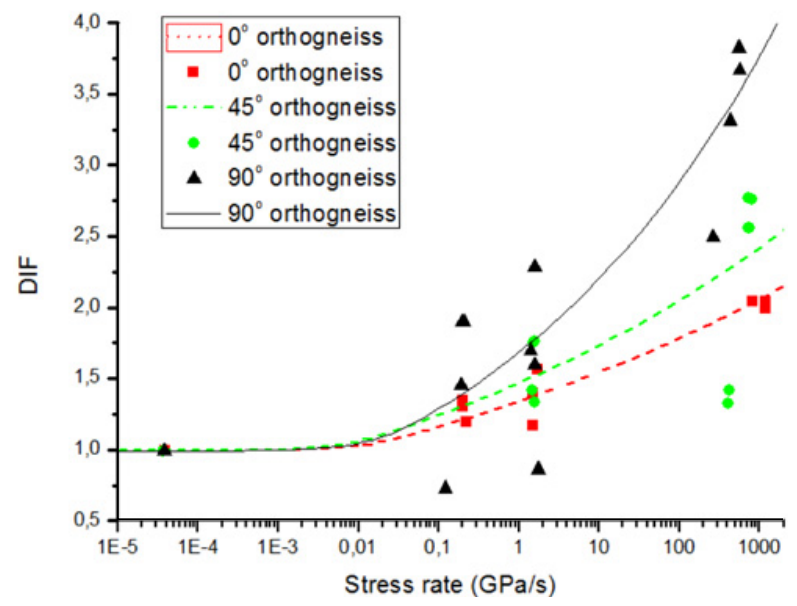

Fig. 2. DIF versus stress rate for the Orthogneiss at $\theta=0^{\circ}$, $\theta=45^{\circ}$ and $\theta=90^{\circ}$.

Table 4. Parameters for equation (7).

\begin{tabular}{|l|c|c|c|}
\hline $\begin{array}{l}\text { Orientation of } \\
\text { schistosity } \\
\left({ }^{\circ}\right)\end{array}$ & $\begin{array}{c}\text { static tensile } \\
\text { stress } \\
(\mathrm{MPa})\end{array}$ & $\begin{array}{c}\tau_{\alpha} \\
(\mu \mathrm{s})\end{array}$ & $\alpha$ \\
\hline 0 & 12,6 & 83 & 15 \\
\hline 45 & 6,1 & 68 & 13 \\
\hline 90 & 2,6 & 24 & 7.6 \\
\hline
\end{tabular}

\subsection{Another approach to the determine dynamic curve}

For better approximation we can consider another criterion. In this case shock deformation can be characterized two major factors: level (amplitude) and speed of loading. This dependence can be expressed by following criterion.

$$
\frac{1}{\tau_{\alpha}} \int_{\mathrm{t}-\tau_{\alpha}}^{\mathrm{t}}\left(\frac{\sigma(\mathrm{s})}{\sigma_{\mathrm{st}}}\right)^{\alpha} \mathrm{ds} \leq 1
$$

where $\tau_{\alpha}$ is the incubation time. Incubation time characterises sensitivity of environment to speed of loading in the given point of environment. The parameter $\alpha$ characterises sensitivity to level (or amplitude) of external force. Usually $\alpha>1$. The parameter $\sigma_{\text {st }}$ is a tensile strength for quasistatic loading and $\sigma(\mathrm{t})$ is the applied stress which for $\mathrm{t}<0$ is supposed to be zero.

On Fig. 2 shows the effect of the orientation on the dynamic tensile strength for different Orientation of schistosity. Points show the experimental data from Tables 1, 2 .

Curves (Fig. 2) show the theoretical line constructed by the formula (7) with parameters from the Table 4. We can see good compliance theoretical approach and experimental data. Parameter $\sigma_{\text {st }}-$ static tensile stress. It is determines the horizontal section on theoretical curve. Parameter $\tau_{\propto}$ can move theoretical curve to the right or to the left. Parameter $\alpha-$ is a determine slope of the curves. 


\section{Conclusion}

Rock tensile strength is stress rate dependent. It has been verified that also for the Orthogneiss this strength increases with the stress rate increasing within the wide stress rate range. The results show a non-linear relationships between strength and stress rates and the influence of the loading direction with respect to the schistosity of the rock examined.

The results compare experimental data and high speed curves confirm application of the concept of incubation time.

\section{Acknowledgements}

This research was financially supported by the RFBR research grant (11-01-00491) and academic program of Presidium of the Russian Academy of Sciences. The research was also supported by the Scientific \& Technological Cooperation Programme Switzerland-Russia in the frame of the Faculty Exchange project "Rate and scale effects in fracture of concrete and rocks".

\section{References}

1. E. Cadoni. Dynamic Characterization of Orthogneiss Rock Subjected to Intermediate and High Strain Rate in Tension. Rock Mech Rock Eng (2010) 43:667-676.

2. G. Barla, N. Innaurato N (1973) Indirect tensile testing of anisotropic rocks. Rock Mech 5:215-230.

3. S. Kubota, Y. Ogata, Y. Wada, G. Simangunsong, H. Shimada, K. Matsui. Estimation of dynamic tensile strength of sandstone. Int J Rock Mech Min Sci (2008) 45:397-406.

4. Petrov, Y.V. "On the quantum" nature of dynamic fracture of brittle solids. Doklady Akademii Nauk, USSR, 321, 66-68, (1991), in Russian.

5. Y. V. Petrov, and N. F. Morozov, On the modeling of fracture of brittle solids. Journal of Applied Mechanics, 61, 710-712 (1994).

6. N. F. Morozov, and Y. V. Petrov, Dynamics of Fracture, Springer, Berlin, 2000.

7. Y. V. Petrov, N. F. Morozov, V. I. Smirnov, Structural Macromechanics Approach in Dynamics of Fracture. Fatigue Fract Engng Mater Struct, 26, 363-372 (2003). 